\title{
FERTILIZAÇÃO DE MUDAS DE ESPÉCIES ARBÓREAS NATIVAS E EXÓTICAS ${ }^{1}$
}

\author{
Sebastião Pires de Moraes Neto ${ }^{2}$, José Leonardo de Moraes Gonçalves ${ }^{3}$, José Carlos Arthur Jr. ${ }^{4}$, Fabiane Ducatti ${ }^{5}$ e \\ José Hamilton Aguirre Jr. ${ }^{6}$
}

\begin{abstract}
RESUMO - Doses crescentes de fertilizante de liberação controlada (FLC) foram comparadas com fertilização convencional e tratamento sem fertilização. Estes tratamentos foram testados em mudas das pioneiras Guazuma ulmifolia (mutambo), Eucalyptus grandis e Pinus caribaea var. caribaea, da secundária inicial Peltophorum dubium (canafístula) e da clímax Calycophyllum spruceanum (mulateiro), as quais cresceram em casa de vegetação. As mudas foram cultivadas em tubetes de $50 \mathrm{~cm}^{3}$ de capacidade, tendo como substrato uma mistura contendo $50 \%$ de húmus de minhoca, $30 \%$ de casca de eucalipto decomposta e $20 \%$ de casca de arroz carbonizada, em base volumétrica. Aos 125 dias após a semeadura, as mudas de G. ulmifolia, E. grandis e P. dubium submetidas à fertilização convencional apresentaram maior crescimento em altura e biomassa seca da parte aérea comparados aos dos tratamentos que receberam FLC. Contudo, para estas espécies, a biomassa seca da raiz das mudas submetidas ao tratamento convencional foram semelhantes à das mudas produzidas com as duas maiores doses de FLC $(4,28$ e $6,42 \mathrm{~kg} / \mathrm{m}^{3}$ de substrato), e a razão entre raiz e parte aérea foi maior para a dose de $6,42 \mathrm{~kg} / \mathrm{m}^{3}$ (FLC), comparada à do tratamento convencional para as duas primeiras espécies. O Calycophyllum spruceanum e o Pinus caribaea var. caribaea tiveram pequeno desenvolvimento em todos os tratamentos, aos 125 dias.
\end{abstract}

Palavras-chave: $\quad$ Mudas, viveiro, fertilizante de liberação controlada e nutrição.

\section{FERTILIZATION OF NATIVE AND EXOTIC TREE SEEDLINGS}

\begin{abstract}
Increasing doses of resin-coated controlled release fertilizers (CRF) were compared with conventional fertilization and treatment without fertilizer. Five species of tree seedlings were used: the pioneers Guazuma ulmifolia (mutambo), Eucalyptus grandis and Pinus caribaea var. caribaea, the early secondary Peltophorum dubium ("canafistula") and the climax Calycophyllum spruceanum ("mulateiro"), grown in greenhouse. The seedlings were tilled in plastic tubes of $50 \mathrm{~cm}^{3}$ capacity, receiving as substrate a mixture of $50 \%$ earthworm humus, 30\% decomposed eucalypt bark, and 20\% carbonized rice husk (by volume). At 125 days after sowing the seedlings of $\boldsymbol{G}$ ulmifolia, $\boldsymbol{E}$. grandis and $\boldsymbol{P}$. dubium submitted to conventional fertilization presented greater growth in height and shoot dry weight (stem plus leaf), in comparison with the CRF treatments. However, for these same species, root dry weight of the seedlings submitted to conventional treatment was not statistically different from that of the seedlings with the two largest doses of CRF (4.28 and $6.42 \mathrm{~kg} / \mathrm{m}^{3}$ of substrate) and the root/shoot ratio was greater for dose $6.42 \mathrm{~kg} / \mathrm{m}^{3}$, compared with the conventional treatment for the two former species. Calycophyllum spruceanum and Pinus caribaea var. caribaea showed little development for all treatments at 125 days.
\end{abstract}

Key words: $\quad$ Seedlings, nursery, controlled-release fertilizer and nutrition.

1 Recebido para publicação em 15.8.2001. Aceito para publicação em 19.2.2003.

2 Dr. em Ciências Biológicas, <spmoraesn@hotmail.com>; ${ }^{3}$ Prof. Dr. do Dep. de Ciências Florestais da Escola Superior de Agricultura "Luiz de Queiroz" - ESALQ-USP, Caixa Postal 9, 13418-900 Piracicaba-SP, <jlmgonca@carpa.ciagri.usp.br>; ${ }^{4}$ Graduando em Engenharia Florestal, ESALQ-USP; ${ }^{5}$ Pós-Graduando em Engenharia Florestal, ESALQ-USP. 


\section{INTRODUÇÃO}

Hoje, devido à grande exploração de florestas nativas realizada em amplas áreas do País, seja para formação de pasto, agricultura, serraria, energia, projetos imobiliários ou para mineração, faz-se mister o replantio de árvores para fins de equilíbrio ambiental e econômico. Este replantio é realizado principalmente através de mudas.

Na produção de mudas, basicamente, são necessários o substrato, o recipiente para acondicioná-lo e o fertilizante. Nos viveiros de alta rotatividade, os produtores, em geral, têm utilizado tubetes de polipropileno de aproximadamente $50 \mathrm{~cm}^{3}$ de capacidade e substratos constituídos principalmente por resíduos orgânicos. Estes substratos são geralmente formados por componentes de microporosidade acima de 50\% em base volumétrica (húmus de minhoca, esterco de gado curtido, casca de eucalipto decomposta), misturados com componentes de macroporosidade entre 25 e $50 \%$ (casca de arroz carbonizada, vermiculita fina, cinza da caldeira de biomassa) (Gonçalves \& Poggiani, 1996).

Para a fertilização, em geral é adicionada ao substrato uma adubação de base com elementos essenciais (macro e micronutrientes), normalmetne na forma sólida, e no decorrer do crescimento das mudas são realizadas fertilizações líquidas com N e K, ou com soluções completas de nutrientes. Geralmente, neste esquema os fertilizantes podem ser solúveis ou pouco solúveis (liberação lenta), porém não são encapsulados.

Os fertilizantes de liberação controlada (FLC), encapsulados ou revestidos com resina (Yague, 1994; Trenkel, 1997), ainda são pouco utilizados no Brasil. Os fertilizantes encapsulados solúveis em água são revestidos por uma capa de resina orgânica permeável à água, normalmente com fertilização NPK, havendo também formulações com micronutrientes. Esta resina de recobrimento controla a liberação de nutrientes. Depois de sua aplicação, a solução do substrato atravessa a camada de resina e dissolve os nutrientes no interior da cápsula, que vão sendo liberados osmoticamente para as mudas, de forma gradual, dependendo da temperatura e umidade do substrato. Em temperaturas mais baixas, diminui a taxa de liberação de nutrientes. Exemplos deste grupo são os fertilizantes Osmocote ${ }^{\circledR}$, Nutricote ${ }^{\circledR}$ e enxofreuréia revestida (Valeri \& Corradini, 2000). Experimentos com adubos encapsulados, em comparação com adubos sem revestimento, mostram que os primeiros resultam

R. Árvore, Viçosa-MG, v.27, n.2, p.129-137, 2003 em maior crescimento e menos lixiviação de nutrientes (Conover \& Poole, 1974; Cole \& Dole, 1997; Huett \& Morris, 1999).

O presente trabalho, visando a produção de mudas arbóreas de melhor qualidade, teve como objetivo comparar o efeito de fertilizante de liberação controlada (FLC) com adubação convencional em mudas de cinco espécies arbóreas.

\section{MATERIAL E MÉTODOS}

O experimento foi realizado em casa de vegetação no Viveiro Florestal do Departamento de Ciências Florestais da Escola Superior de Agricultura 'Luiz de Queiroz', Piracicaba - SP. Na casa de vegetação, durante o período experimental, a média das temperaturas máximas \pm desvio-padrão foi $31,0 \pm 1,0{ }^{\circ} \mathrm{C}$ e a média das temperaturas mínimas foi $17,2 \pm 2,4^{\circ} \mathrm{C}$, utilizando como base de cálculo as médias mensais.

Os tratamentos de fertilização foram:

- Tratamento 1: testemunha - sem fertilizante no substrato;

- Tratamento 2: fertilização de liberação controlada (FLC) na dose de $2,14 \mathrm{~kg} / \mathrm{m}^{3}$ de substrato;

- Tratamento 3: FLC na dose de 4,28 kg/m³ de substrato;

- Tratamento 4: FLC na dose de $6,42 \mathrm{~kg} / \mathrm{m}^{3}$ de substrato; e

- Tratamento 5: fertilização convencional.

Para cada uma das espécies, cujas características estão descritas no Quadro 1, foi instalado um ensaio, constituído pelos cinco tratamentos descritos, dispostos em blocos ao acaso, com quatro repetições, sendo cada parcela composta por 48 mudas.

Como fertilizante de liberação controlada (FLC) utilizou-se o Osmocote ${ }^{\circledR} 14 \% \mathrm{~N}-14 \% \mathrm{P}_{2} \mathrm{O}_{5}-14 \% \mathrm{~K}_{2} \mathrm{O}$, na forma granular, revestido com uma resina orgânica semipermeável. Segundo as especificações técnicas, quando colocado em substrato úmido a uma temperatura média de $21,1{ }^{\circ} \mathrm{C}$, libera todos nutrientes entre três a quatro meses. A composição do FLC utilizado possui os seguintes nutrientes: nitrato de amônio, sulfato de potássio, fosfatos de amônio, fosfatos de cálcio e fluoreto de cálcio. O N é constituído em $8,2 \%$ na forma amoniacal e 5,8\% na forma nítrica.

A adubação de base do tratamento 5 (convencional) consistiu na mistura de 200 g FTE BR12 + 500 g de 
sulfato de amônio $+1,5 \mathrm{~kg}$ de superfosfato simples + $150 \mathrm{~g} \mathrm{KCl}$ por $\mathrm{m}^{3}$ de substrato. Já, a fertilização de cobertura consistiu basicamente da aplicação de sulfato de amônio (S.A.) a cada duas semanas na concentração de $10 \mathrm{~g}$ de S.A. por litro de água, após um mês da germinação das sementes. A quantidade de 2,5 1 desta solução serve para adubar aproximadamente 100 mudas, usando regador. Na primeira adubação de cobertura fez-se uma mistura de S.A., na mesma concentração citada anteriormente, com $\mathrm{KCl}$ na concentração de $3 \mathrm{~g}$ por litro de água.

As mudas foram produzidas em tubetes de $50 \mathrm{~cm}^{3}$ de volume útil ( $12 \mathrm{~cm}$ de comprimento, $3 \mathrm{~cm}$ de diâmetro superior e $1 \mathrm{~cm}$ de diâmetro na parte inferior), as quais foram acondicionadas em bandejas de isopor com 96 aberturas. O substrato utilizado foi a mistura de $50 \%$ de húmus de minhoca, $30 \%$ de casca de eucalipto decomposta e $20 \%$ de casca de arroz carbonizada, em base volumétrica. A semeadura das cinco espécies foi feita diretamente nos tubetes em 29/6/1998, e o período experimental durou 125 dias.

Foram realizadas três medições em altura e diâmetro do coleto durante o período experimental, a partir de 65 dias após a semeadura. O material vegetal das mudas das cinco espécies para todos os tratamentos, após o término do experimento, foi dividido em parte aérea (folha mais caule) e raiz e, em seguida, colocado em estufa a $65^{\circ} \mathrm{C}$, até atingir peso constante. Após a pesagem, a parte aérea das mudas de Guazuma ulmifolia, Peltophorum dubium e Eucalyptus grandis foi moída para determinação da concentração de macro e micronutrientes por digestão nitricoperclórica. A determinação de $\mathrm{N}$ foi realizada pelo método de Kjeldahl (Sarruge \& Haag, 1974). As mudas de Calycophyllum spruceanum e de Pinus caribaea var. caribaea não foram submetidas a determinações nutricionais, por apresentarem tamanho diminuto ao final do experimento.

A análise estatística utilizou o programa SAS (SAS Institute Inc., 1990) para a ANOVA e o teste de Tukey para comparação entre médias (Steel \& Torrie, 1980). Quando necessário, foram feitas transformações dos dados para homogeneizar as variâncias e adequá-los à distribuição normal.

\section{RESULTADOS E DISCUSSÃO}

O incremento em altura para a Guazuma ulmifolia, o Peltophorum dubium e o Eucalyptus grandis evidenciou resposta similar em relação aos tratamentos aplicados, porém com amplitudes diferentes, em que o tratamento convencional (tratamento 5) destacou-se dos demais aos 125 dias pós-semeadura (Figura 1). Para o Eucalyptus grandis, o diferencial entre o tratamento com maior dose de FLC e o com dose intermediária de FLC foi mais pronunciado do que para as outras duas espécies. Quanto ao Calycophyllum spruceanum e o Pinus caribaea var. caribaea (Figura 1), houve tendência de maior incremento em altura dos 95 aos 125 dias nos dois tratamentos com maiores doses de FLC junto com o tratamento convencional, em relação aos outros dois tratamentos para a primeira espécie, e maior incremento das mudas no tratamento convencional em relação aos demais tratamentos para a segunda espécie.

O diâmetro do coleto no tratamento com maior dose de FLC e no convencional não diferiu para a Guazuma ulmifolia, o Peltophorum dubium e o Calycophyllum spruceanum. Para o Eucalyptus grandis e Pinus caribaea var. caribaea, o tratamento convencional resultou em

Quadro 1 - Características das espécies utilizadas

Table 1 - Characteristics of the species used

\begin{tabular}{|l|l|l|c|c|c|}
\hline \multicolumn{1}{|c|}{ Espécie } & Família & Procedência & $\begin{array}{c}\text { Peso da } \\
\text { semente } \\
\text { (mg) }\end{array}$ & $\begin{array}{c}\text { Dispersão } \\
\text { da semente }\end{array}$ & $\begin{array}{c}\text { Categoria } \\
\text { sucessional* }\end{array}$ \\
\hline Guazuma ulmifolia Lam. & Sterculiaceae & Alto Juruá-AC & 6 & zoocórica & pioneira \\
\hline Peltophorum dubium (Spreng.) Taub. & $\begin{array}{l}\text { Leguminosae } \\
\text { Cesalpinoideae }\end{array}$ & Piracicaba-SP & 50 & anemocórica & $\begin{array}{l}\text { secundária } \\
\text { inicial }\end{array}$ \\
\hline Calycophyllum spruceanum (Benth.) K. Schum. & Rubiaceae & Alto Juruá-AC & 0,15 & anemocórica & clímax \\
\hline Pinus caribaea Morelet var. caribaea & Pinaceae & Aracruz-ES & 17 & anemocórica & pioneira \\
\hline Eucalyptus grandis W. Hill ex Maiden & Myrtaceae & Anhembi-SP & 0,27 & autocórica & pioneira \\
\hline
\end{tabular}

* Categoria sucessional baseada em Budowsky (1965). 
Guazuma ulmifolia

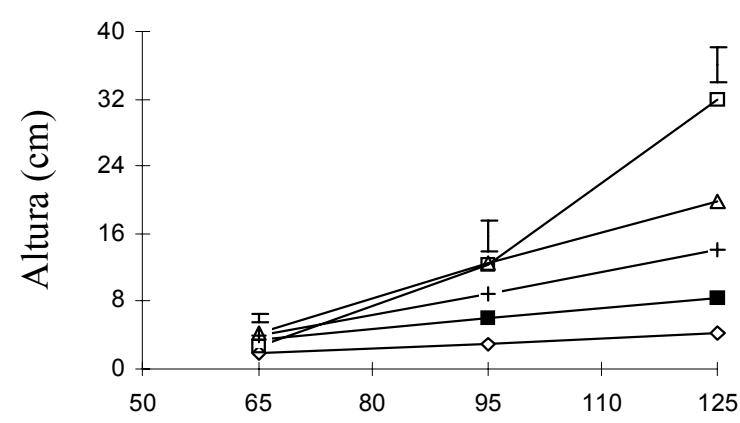

Calycophyllum spruceanum

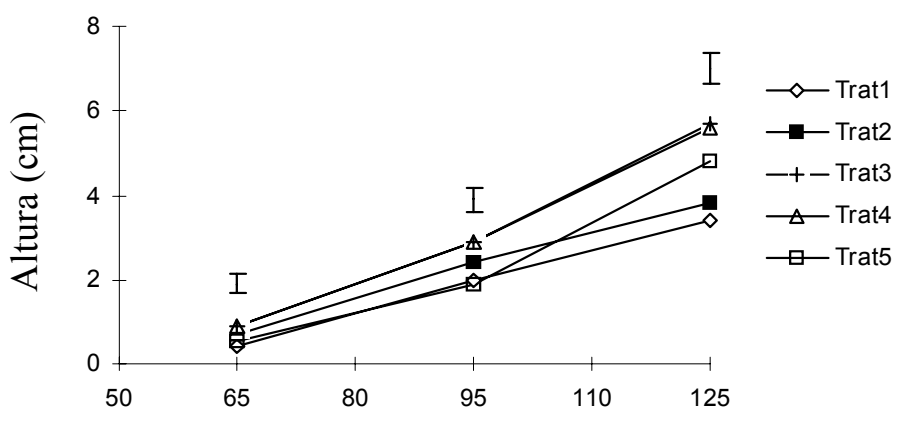

Eucalyptus grandis

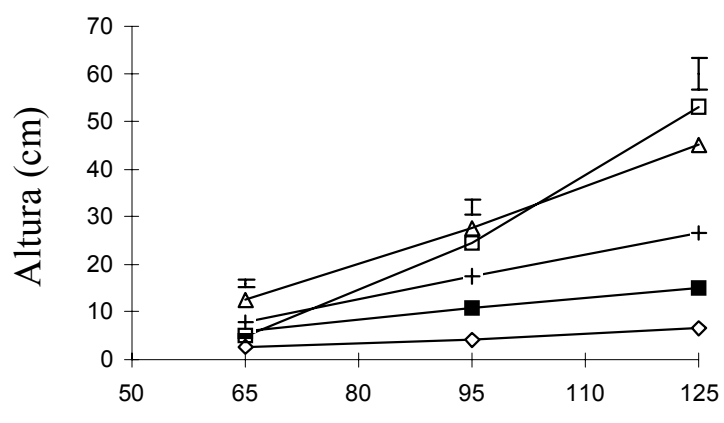

Tempo Após a Semeadura (dias)
Peltophorum dubium

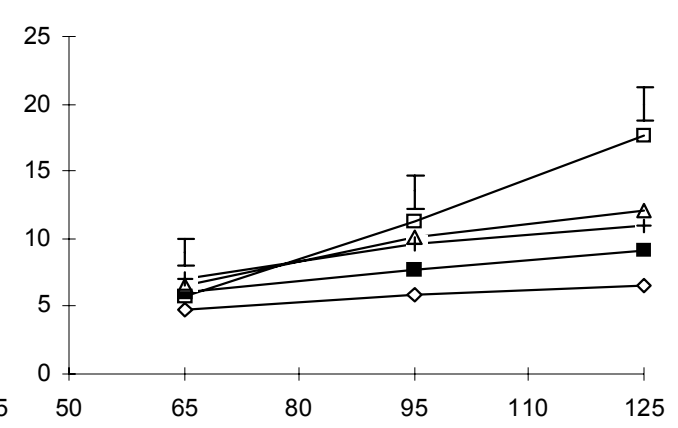

Pinus caribaea var. caribaea

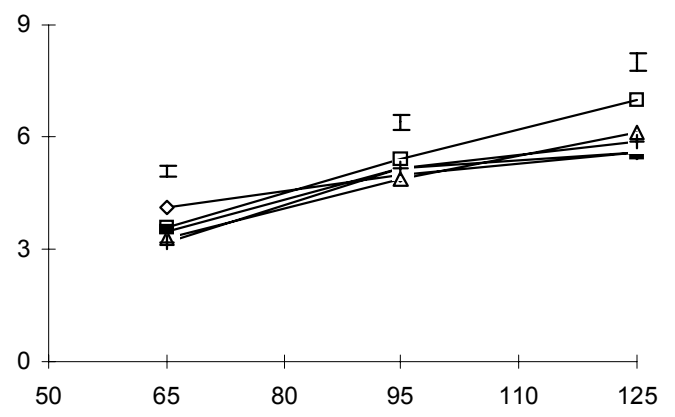

Trat1 = testemunha, sem fertilização; Trat2 =2,14 kg/m³ de Osm.; Trat3=4,28 kg/m³ de Osm.; Trat4 =6,42 kg/m³ de Osm.; e Trat5 = fertilização convencional.

Figura 1 - Altura das mudas em função do tempo e dos tratamentos. As barras verticais representam as diferenças mínimas significativas $(\mathrm{P} \leq 0,05)$

Figure 1 - Height of seedlings in function of time and treatments. The vertical bars represent the least significant differences $(P \leq 0,05)$.

R. Árvore, Viçosa-MG, v.27, n.2, p.129-137, 2003 
mudas com diâmetro maior que nos demais tratamentos (Quadro 2). Para biomassa seca da parte aérea (BSPA), o tratamento convencional resultou em mudas com maior valor em relação aos demais tratamentos para todas as espécies, com exceção do C. spruceanum, em que a BSPA das mudas no tratamento com maior dose de FLC foi maior do que no tratamento convencional. Quanto à biomassa seca da raiz (BSR), o tratamento com maior dose de FLC e o convencional resultaram em maiores valores do que os demais tratamentos, com exceção de o C. spruceanum, em que a BSR das mudas com maior dose de FLC foi maior do que o tratamento convencional.
Para biomassa seca total (BST), as mudas no tratamento convencional mostraram maiores valores do que os outros tratamentos para o Peltophorum dubium, o Eucalyptus grandis e o Pinus caribaea var. caribaea (Quadro 2), e as mudas de Guazuma ulmifolia não diferiram entre este tratamento e o com maior dose de FLC. Já para Calycophyllum spruceanum, os tratamentos que resultaram em maiores valores de BST foram os com dose maior e intermediária de FLC. Quanto à razão entre a raiz e a parte aérea $(\mathrm{R} / \mathrm{P})$, a testemunha e o tratamento de menor dose de FLC (tratamento 2) não diferiram para todas as espécies. Os menores valores absolutos da razão $\mathrm{R} / \mathrm{P}$ ocorreram no tratamento convencional.

Quadro 2 - Dimensões de algumas variáveis da parte aérea e sistema radicular de três espécies, em função dos tratamentos aplicados aos 125 dias após a semeadura

Table 2 - Dimensions of some shoot and root variables of three species, in function of the applied treatments at 125 days after sowing

\begin{tabular}{|c|c|c|c|c|c|c|}
\hline \multirow[b]{2}{*}{ Tratamento } & \multirow{2}{*}{$\begin{array}{l}\text { Diâmetro do } \\
\text { Coleto } \\
\text { (mm) }\end{array}$} & \multirow{2}{*}{$\begin{array}{c}\text { Altura } \\
(\mathrm{cm})\end{array}$} & \multicolumn{3}{|c|}{ Biomassa Seca $(\mathrm{g})$} & \multirow{2}{*}{$\begin{array}{l}\text { Raiz } \\
\text { (PA) }\end{array}$} \\
\hline & & & $\begin{array}{c}\text { Parte aérea } \\
\text { (PA) }\end{array}$ & Raiz & Total & \\
\hline \multicolumn{7}{|c|}{ Guazuma ulmifolia (mutambo) } \\
\hline 1 & $2,0 \mathrm{D}$ & $4,3 \mathrm{D}$ & $0,09 \mathrm{D}$ & $0,10 \mathrm{~B}$ & $0,19 \mathrm{D}$ & $1,13 \mathrm{~A}$ \\
\hline 2 & $2,9 \mathrm{C}$ & $8,4 \mathrm{D}$ & $0,27 \mathrm{CD}$ & $0,35 \mathrm{~A}$ & $0,62 \mathrm{CD}$ & $1,28 \mathrm{~A}$ \\
\hline 3 & $3,3 \mathrm{~B}$ & $14,1 \mathrm{C}$ & $0,42 \mathrm{C}$ & $0,44 \mathrm{~A}$ & $0,86 \mathrm{BC}$ & $1,09 \mathrm{~A}$ \\
\hline 4 & $3,8 \mathrm{~A}$ & $19,8 \mathrm{~B}$ & $0,74 \mathrm{~B}$ & $0,56 \mathrm{~A}$ & $1,30 \mathrm{AB}$ & $0,79 \mathrm{~B}$ \\
\hline 5 & $3,9 \mathrm{~A}$ & $31,9 \mathrm{~A}$ & $1,11 \mathrm{~A}$ & $0,38 \mathrm{~A}$ & $1,49 \mathrm{~A}$ & $0,37 \mathrm{C}$ \\
\hline \multicolumn{7}{|c|}{ Peltophorum dubium (canafistula) } \\
\hline 1 & $2,0 \mathrm{C}$ & $6,6 \mathrm{D}$ & $0,21 \mathrm{D}$ & $0,11 \mathrm{C}$ & $0,33 \mathrm{D}$ & $0,52 \mathrm{~A}$ \\
\hline 2 & $2,6 \mathrm{~B}$ & $9,1 \mathrm{C}$ & $0,50 \mathrm{C}$ & $0,23 \mathrm{~B}$ & $0,63 \mathrm{C}$ & $0,46 \mathrm{~A}$ \\
\hline 3 & $3,3 \mathrm{~A}$ & $11,0 \mathrm{BC}$ & $0,70 \mathrm{BC}$ & $0,30 \mathrm{AB}$ & $1,00 \mathrm{BC}$ & $0,44 \mathrm{AB}$ \\
\hline 4 & $3,5 \mathrm{~A}$ & $12,1 \mathrm{~B}$ & $0,92 \mathrm{~B}$ & $0,32 \mathrm{~A}$ & $1,24 \mathrm{~B}$ & $0,37 \mathrm{~B}$ \\
\hline 5 & $3,9 \mathrm{~A}$ & $17,7 \mathrm{~A}$ & $1,69 \mathrm{~A}$ & $0,30 \mathrm{AB}$ & $1,99 \mathrm{~A}$ & $0,17 \mathrm{C}$ \\
\hline \multicolumn{7}{|c|}{ Calycophyllum spruceanum } \\
\hline 1 & $1,7 \mathrm{C}$ & $3,4 \mathrm{C}$ & $0,10 \mathrm{C}$ & $0,05 \mathrm{~B}$ & $0,15 \mathrm{C}$ & $0,50 \mathrm{~A}$ \\
\hline 2 & $1,5 \mathrm{D}$ & $3,8 \mathrm{C}$ & $0,11 \mathrm{C}$ & $0,05 \mathrm{~B}$ & $0,18 \mathrm{C}$ & $0,45 \mathrm{~A}$ \\
\hline 3 & $2,2 \mathrm{~A}$ & $5,7 \mathrm{~A}$ & $0,26 \mathrm{~A}$ & $0,08 \mathrm{~A}$ & $0,34 \mathrm{~A}$ & $0,31 \mathrm{~B}$ \\
\hline 4 & $2,1 \mathrm{~B}$ & $5,6 \mathrm{~A}$ & $0,27 \mathrm{~A}$ & $0,08 \mathrm{~A}$ & $0,35 \mathrm{~A}$ & $0,30 \mathrm{~B}$ \\
\hline 5 & $1,9 \mathrm{~B}$ & $4,8 \mathrm{~B}$ & $0,22 \mathrm{~B}$ & $0,05 \mathrm{~B}$ & $0,27 \mathrm{~B}$ & $0,23 \mathrm{C}$ \\
\hline \multicolumn{7}{|c|}{ Eucalyptus grandis } \\
\hline 1 & $1,1 \mathrm{D}$ & $6,6 \mathrm{E}$ & $0,10 \mathrm{E}$ & $0,05 \mathrm{D}$ & $0,15 \mathrm{E}$ & $0,49 \mathrm{~A}$ \\
\hline 2 & $1,9 \mathrm{C}$ & $15,1 \mathrm{D}$ & $0,45 \mathrm{D}$ & $0,16 \mathrm{C}$ & $0,61 \mathrm{D}$ & $0,43 \mathrm{AB}$ \\
\hline 3 & $2,1 \mathrm{C}$ & $26,6 \mathrm{C}$ & $0,89 \mathrm{C}$ & $0,26 \mathrm{~B}$ & $1,15 \mathrm{C}$ & $0,33 \mathrm{BC}$ \\
\hline 4 & $2,8 \mathrm{~B}$ & $45,2 \mathrm{~B}$ & $1,52 \mathrm{~B}$ & $0,34 \mathrm{~A}$ & $1,86 \mathrm{~B}$ & $0,24 \mathrm{CD}$ \\
\hline 5 & $3,3 \mathrm{~A}$ & $53,1 \mathrm{~A}$ & $1,89 \mathrm{~A}$ & $0,31 \mathrm{AB}$ & $2,20 \mathrm{~A}$ & $0,19 \mathrm{D}$ \\
\hline \multicolumn{7}{|c|}{ Pinus caribaea var. caribaea } \\
\hline 1 & $1,2 \mathrm{D}$ & $5,6 \mathrm{C}$ & $0,11 \mathrm{D}$ & $0,06 \mathrm{C}$ & $0,17 \mathrm{E}$ & $0,54 \mathrm{~A}$ \\
\hline 2 & $1,4 \mathrm{BC}$ & $5,6 \mathrm{C}$ & $0,14 \mathrm{C}$ & $0,07 \mathrm{BC}$ & $0,21 \mathrm{D}$ & $0,50 \mathrm{AB}$ \\
\hline 3 & $1,4 \mathrm{C}$ & $5,9 \mathrm{BC}$ & $0,18 \mathrm{BC}$ & $0,08 \mathrm{~B}$ & $0,26 \mathrm{BC}$ & $0,44 \mathrm{BC}$ \\
\hline 4 & $1,5 \mathrm{~B}$ & $6,1 \mathrm{~B}$ & $0,21 \mathrm{~B}$ & $0,09 \mathrm{AB}$ & $0,30 \mathrm{~B}$ & $0,43 \mathrm{BC}$ \\
\hline 5 & $2,0 \mathrm{~A}$ & $7,0 \mathrm{~A}$ & $0,29 \mathrm{~A}$ & $0,11 \mathrm{~A}$ & $0,40 \mathrm{~A}$ & $0,38 \mathrm{C}$ \\
\hline
\end{tabular}

As médias com as mesmas letras dentro de cada espécie não diferem estatisticamente (teste de Tukey, $\mathrm{P}>0,05$ ).

Means with the same letter are not significantly different within each species (Tukey test, $P>0,05$ ).

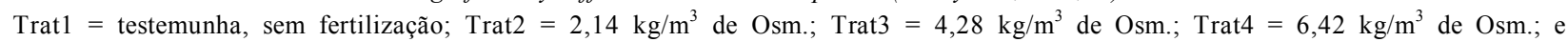
Trat5 = fertilização convencional. 
A concentração de nutrientes da parte aérea das mudas de três espécies é apresentada no Quadro 3. Observa-se, de modo geral, que tanto para os macronutrientes quanto para os micronutrientes não houve grande variação da concentração de nutrientes entre os tratamentos. Contudo, houve casos de diferenças entre tratamentos. Assim, nas concentrações de N, P e Ca para a Guazuma ulmifolia, a testemunha mostrou maior valor que o tratamento convencional. Quanto ao K, os tratamentos com dose intermediária e maior de FLC apresentaram maiores valores do que o convencional. Para o $\mathrm{Mg}$, a concentração da parte aérea das mudas da testemunha foi maior que a do tratamento com dose intermediária de FLC, enquanto para o $\mathrm{Zn}$ o valor no tratamento convencional foi maior que o com dose intermediária de FLC. Já para o $\mathrm{S}, \mathrm{Fe}, \mathrm{Cu}$ e $\mathrm{B}$, não houve diferença entre os tratamentos.

Para o Peltophorum dubium, a concentração de N na parte aérea das mudas foi maior no tratamento convencional que nos tratamentos com dose menor e intermediária de FLC. Quanto ao P e Fe, a concentração da parte aérea das mudas da testemunha foi maior do que a dos tratamentos com dose intermediária e maior de FLC. Para o Mn, o valor no tratamento convencional foi maior que nos demais tratamentos. Quanto aos elementos $\mathrm{K}, \mathrm{Ca}$, $\mathrm{Mg}, \mathrm{S}, \mathrm{Cu}, \mathrm{Zn}$ e B, não houve diferença entre os tratamentos.

Para o Eucalyptus grandis (Quadro 3), a concentração de $\mathrm{N}$ e Fe na parte aérea das mudas mostrou maior valor para o tratamento convencional do que para a testemunha. Quanto ao B, a testemunha mostrou maior valor que os tratamentos com as maiores doses de FLC. Para os elementos $\mathrm{P}, \mathrm{K}, \mathrm{Ca}, \mathrm{Mg}, \mathrm{S}, \mathrm{Cu}, \mathrm{Mn}$ e $\mathrm{Zn}$, não houve diferença entre os tratamentos.

Quanto ao conteúdo de nutrientes da parte aérea das mudas (Quadro 4), observa-se, de modo geral, uma progressão de valores da testemunha em direção ao tratamento convencional para as três espécies, porém nem sempre houve diferença entre um e outro tratamento. Para a Guazuma ulmifolia, o conteúdo dos elementos P e K não diferiram entre os tratamentos convencional e o com

Quadro 3 - Concentração de nutrientes da parte aérea (caule mais folha) das mudas Table 3 -Shoot (stem plus leaf) nutrient concentration of the seedlings

\begin{tabular}{|c|c|c|c|c|c|c|c|c|c|c|c|}
\hline \multirow{2}{*}{$\begin{array}{l}\text { Trata- } \\
\text { mento }\end{array}$} & \multicolumn{6}{|c|}{ Macronutriente $(\mathrm{g} / \mathrm{kg})$} & \multicolumn{5}{|c|}{ Micronutriente $(\mathrm{mg} / \mathrm{kg})$} \\
\hline & $\mathrm{N}$ & $\mathrm{P}$ & $\mathrm{K}$ & $\mathrm{Ca}$ & $\mathrm{Mg}$ & $\mathrm{S}$ & $\mathrm{Fe}$ & $\mathrm{Cu}$ & $\mathrm{Mn}$ & $\mathrm{Zn}$ & $\mathrm{B}$ \\
\hline \multicolumn{12}{|c|}{ Guazuma ulmifolia } \\
\hline 1 & $13 \mathrm{a}$ & $5,9 \mathrm{a}$ & $15 \mathrm{ab}$ & $25 \mathrm{a}$ & $9,3 \mathrm{a}$ & $2,5 \mathrm{a}$ & $318 \mathrm{a}$ & $16 \mathrm{a}$ & $53 \mathrm{c}$ & $30 \mathrm{ab}$ & $77 \mathrm{a}$ \\
\hline 2 & $11 \mathrm{ab}$ & $4,3 \mathrm{~b}$ & $16 \mathrm{ab}$ & $22 \mathrm{ab}$ & $9,0 \mathrm{a}$ & $2,3 \mathrm{a}$ & $320 \mathrm{a}$ & $11 \mathrm{a}$ & $103 \mathrm{~b}$ & $29 \mathrm{ab}$ & $89 \mathrm{a}$ \\
\hline 3 & $10 \mathrm{bc}$ & $4,1 \mathrm{~b}$ & $17 \mathrm{a}$ & $18 \mathrm{~b}$ & $7,4 \mathrm{~b}$ & $2,2 \mathrm{a}$ & $347 \mathrm{a}$ & $15 \mathrm{a}$ & $170 \mathrm{a}$ & $27 \mathrm{~b}$ & $68 \mathrm{a}$ \\
\hline 4 & $10 \mathrm{bc}$ & $3,3 \mathrm{~b}$ & $19 \mathrm{a}$ & $18 \mathrm{~b}$ & $8,1 \mathrm{ab}$ & $2,1 \mathrm{a}$ & $347 \mathrm{a}$ & $15 \mathrm{a}$ & $160 \mathrm{a}$ & $28 \mathrm{ab}$ & $62 \mathrm{a}$ \\
\hline 5 & $9 \mathrm{c}$ & $1,8 \mathrm{c}$ & $13 \mathrm{~b}$ & $19 \mathrm{~b}$ & $7,8 \mathrm{ab}$ & $2,3 \mathrm{a}$ & $307 \mathrm{a}$ & $15 \mathrm{a}$ & $50 \mathrm{c}$ & $33 \mathrm{a}$ & $63 \mathrm{a}$ \\
\hline \multicolumn{12}{|c|}{ Peltophorum dubium } \\
\hline 1 & $10 \mathrm{ab}$ & $2,7 \mathrm{a}$ & $11 \mathrm{a}$ & $13 \mathrm{a}$ & $1,9 \mathrm{a}$ & $2,1 \mathrm{a}$ & $380 \mathrm{a}$ & $17 \mathrm{a}$ & $67 \mathrm{c}$ & $38 \mathrm{a}$ & $36 \mathrm{a}$ \\
\hline 2 & $9 \mathrm{~b}$ & $1,9 \mathrm{~b}$ & $11 \mathrm{a}$ & $10 \mathrm{a}$ & $1,6 \mathrm{a}$ & $1,8 \mathrm{a}$ & $287 \mathrm{ab}$ & $14 \mathrm{a}$ & $57 \mathrm{c}$ & $35 \mathrm{a}$ & $44 \mathrm{a}$ \\
\hline 3 & $9 \mathrm{~b}$ & $2,1 \mathrm{~b}$ & $12 \mathrm{a}$ & $11 \mathrm{a}$ & $1,5 \mathrm{a}$ & $1,8 \mathrm{a}$ & $230 \mathrm{~b}$ & $13 \mathrm{a}$ & $63 \mathrm{c}$ & $38 \mathrm{a}$ & $41 \mathrm{a}$ \\
\hline 4 & $11 \mathrm{ab}$ & $2,0 \mathrm{~b}$ & $11 \mathrm{a}$ & $11 \mathrm{a}$ & $1,7 \mathrm{a}$ & $2,1 \mathrm{a}$ & $273 \mathrm{~b}$ & $16 \mathrm{a}$ & $90 \mathrm{~b}$ & $35 \mathrm{a}$ & $38 \mathrm{a}$ \\
\hline 5 & $15 \mathrm{a}$ & $1,8 \mathrm{~b}$ & $9 \mathrm{a}$ & $11 \mathrm{a}$ & $1,9 \mathrm{a}$ & $2,5 \mathrm{a}$ & $313 \mathrm{ab}$ & $15 \mathrm{a}$ & $120 \mathrm{a}$ & $40 \mathrm{a}$ & $36 \mathrm{a}$ \\
\hline \multicolumn{12}{|c|}{ Eucalyptus grandis } \\
\hline 1 & $8 \mathrm{~b}$ & $2,8 \mathrm{a}$ & $19 \mathrm{a}$ & $14 \mathrm{a}$ & $7,6 \mathrm{a}$ & $1,1 \mathrm{a}$ & $177 \mathrm{~b}$ & $12 \mathrm{a}$ & $477 \mathrm{a}$ & $38 \mathrm{a}$ & $51 \mathrm{a}$ \\
\hline 2 & $7 \mathrm{~b}$ & $2,6 \mathrm{a}$ & $19 \mathrm{a}$ & $16 \mathrm{a}$ & $7,3 \mathrm{a}$ & $0,9 \mathrm{a}$ & $197 \mathrm{ab}$ & $14 \mathrm{a}$ & $483 \mathrm{a}$ & $38 \mathrm{a}$ & $38 \mathrm{ab}$ \\
\hline 3 & $7 \mathrm{~b}$ & $2,2 \mathrm{a}$ & $19 \mathrm{a}$ & $14 \mathrm{a}$ & $7,0 \mathrm{a}$ & $0,7 \mathrm{a}$ & $190 \mathrm{ab}$ & $15 \mathrm{a}$ & $503 \mathrm{a}$ & $38 \mathrm{a}$ & $34 \mathrm{~b}$ \\
\hline 4 & $7 \mathrm{~b}$ & $2,2 \mathrm{a}$ & $20 \mathrm{a}$ & $16 \mathrm{a}$ & $3,9 \mathrm{a}$ & $0,6 \mathrm{a}$ & $223 \mathrm{ab}$ & $18 \mathrm{a}$ & $550 \mathrm{a}$ & $32 \mathrm{a}$ & $25 \mathrm{~b}$ \\
\hline 5 & $11 \mathrm{a}$ & $2,1 \mathrm{a}$ & $19 \mathrm{a}$ & $15 \mathrm{a}$ & $4,6 \mathrm{a}$ & $0,8 \mathrm{a}$ & $233 \mathrm{a}$ & $15 \mathrm{a}$ & $547 \mathrm{a}$ & $32 \mathrm{a}$ & $39 \mathrm{ab}$ \\
\hline
\end{tabular}

As médias com as mesmas letras dentro de cada espécie não diferem estatisticamente (teste deTukey, P>0,05).

Means with the same letter are not significantly different within each species (Tukey test, P>0,05).

Trat1 = testemunha, sem fertilização; Trat2 = 2,14 kg/m $\mathrm{m}^{3}$ de Osm.; Trat3 = 4,28 kg/m $\mathrm{m}^{3} \mathrm{de}$ Osm.; Trat4 = 6,42 kg/m $\mathrm{m}^{3}$ de Osm.; e Trat5 $=$ fertilização convencional.

R. Árvore, Viçosa-MG, v.27, n.2, p.129-137, 2003 
Quadro 4 - Conteúdo de nutrientes da parte aérea (caule mais folha) das mudas Table 4-Shoot (stem plus leaf) nutrient content of the seedlings

\begin{tabular}{|c|c|c|c|c|c|c|c|c|c|c|c|}
\hline \multirow{2}{*}{$\begin{array}{l}\text { Trata- } \\
\text { mento }\end{array}$} & \multicolumn{6}{|c|}{ Macronutriente (mg/planta) } & \multicolumn{5}{|c|}{ Micronutriente $(\mu \mathrm{g} / \mathrm{planta})$} \\
\hline & $\mathrm{N}$ & $\mathrm{P}$ & $\mathrm{K}$ & $\mathrm{Ca}$ & $\mathrm{Mg}$ & $\mathrm{S}$ & $\mathrm{Fe}$ & $\mathrm{Cu}$ & $\mathrm{Mn}$ & $\mathrm{Zn}$ & $\mathrm{B}$ \\
\hline \multicolumn{12}{|c|}{ Guazuma ulmifolia } \\
\hline 1 & $1 \mathrm{~d}$ & $0,5 \mathrm{~d}$ & $1 \mathrm{~d}$ & $2 \mathrm{~d}$ & $0,8 \mathrm{e}$ & $0,2 \mathrm{~d}$ & $28 \mathrm{~d}$ & $1 \mathrm{~d}$ & $5 \mathrm{~d}$ & $3 \mathrm{~d}$ & $7 \mathrm{~d}$ \\
\hline 2 & $3 \mathrm{c}$ & $1,2 \mathrm{~cd}$ & $4 \mathrm{c}$ & $6 \mathrm{c}$ & $2,4 \mathrm{~d}$ & $0,6 \mathrm{c}$ & $86 \mathrm{~cd}$ & $3 \mathrm{~cd}$ & $28 \mathrm{c}$ & $8 \mathrm{~cd}$ & $24 \mathrm{c}$ \\
\hline 3 & $4 \mathrm{c}$ & $1,7 \mathrm{bc}$ & $7 \mathrm{~b}$ & $7 \mathrm{c}$ & $3,1 \mathrm{c}$ & $0,9 \mathrm{c}$ & $146 \mathrm{c}$ & $6 \mathrm{c}$ & $71 \mathrm{~b}$ & $11 \mathrm{c}$ & $29 \mathrm{c}$ \\
\hline 4 & $7 \mathrm{~b}$ & $2,4 \mathrm{a}$ & $14 \mathrm{a}$ & $14 \mathrm{~b}$ & $6,0 \mathrm{~b}$ & $1,5 \mathrm{~b}$ & $256 \mathrm{~b}$ & $11 \mathrm{~b}$ & $118 \mathrm{a}$ & $21 \mathrm{~b}$ & $46 \mathrm{~b}$ \\
\hline 5 & $10 \mathrm{a}$ & $2,0 \mathrm{ab}$ & $14 \mathrm{a}$ & $21 \mathrm{a}$ & $8,7 \mathrm{a}$ & $2,5 \mathrm{a}$ & $340 \mathrm{a}$ & $16 \mathrm{a}$ & $55 \mathrm{~b}$ & $37 \mathrm{a}$ & $70 \mathrm{a}$ \\
\hline \multicolumn{12}{|c|}{ Peltophorum dubium } \\
\hline 1 & $2 \mathrm{~d}$ & $0,6 \mathrm{~d}$ & $2 \mathrm{~d}$ & $3 \mathrm{~d}$ & $0,4 \mathrm{~d}$ & $0,4 \mathrm{~d}$ & $80 \mathrm{c}$ & $3 \mathrm{c}$ & $14 \mathrm{~d}$ & $8 \mathrm{~d}$ & $8 \mathrm{c}$ \\
\hline 2 & $5 \mathrm{c}$ & $0,9 \mathrm{c}$ & $6 \mathrm{~cd}$ & $5 \mathrm{~cd}$ & $0,8 \mathrm{~cd}$ & $0,9 \mathrm{~cd}$ & $143 \mathrm{c}$ & $7 \mathrm{c}$ & $28 \mathrm{~cd}$ & $18 \mathrm{c}$ & $22 b$ \\
\hline 3 & $7 \mathrm{c}$ & $1,4 \mathrm{~b}$ & $8 \mathrm{bc}$ & $8 \mathrm{bc}$ & $1,1 \mathrm{bc}$ & $1,2 \mathrm{bc}$ & $161 \mathrm{c}$ & $9 \mathrm{bc}$ & $44 \mathrm{c}$ & $27 \mathrm{~b}$ & $28 \mathrm{~b}$ \\
\hline 4 & $10 \mathrm{~b}$ & $1,8 \mathrm{~b}$ & $10 \mathrm{~b}$ & $10 \mathrm{~b}$ & $1,5 \mathrm{~b}$ & $1,9 \mathrm{~b}$ & $251 \mathrm{~b}$ & $15 \mathrm{~b}$ & $83 \mathrm{~b}$ & $32 \mathrm{~b}$ & $35 \mathrm{~b}$ \\
\hline 5 & $25 \mathrm{a}$ & $3,0 \mathrm{a}$ & $16 \mathrm{a}$ & $19 \mathrm{a}$ & $3,3 \mathrm{a}$ & $4,2 \mathrm{a}$ & $529 \mathrm{a}$ & $26 \mathrm{a}$ & $203 \mathrm{a}$ & $68 \mathrm{a}$ & $61 \mathrm{a}$ \\
\hline \multicolumn{12}{|c|}{ Eucalyptus grandis } \\
\hline 1 & $1 \mathrm{e}$ & $0,3 \mathrm{c}$ & $2 \mathrm{e}$ & $1 \mathrm{~d}$ & $0,8 \mathrm{~b}$ & $0,1 \mathrm{~d}$ & $18 \mathrm{~d}$ & $1 \mathrm{c}$ & $48 \mathrm{e}$ & $4 d$ & $5 \mathrm{~d}$ \\
\hline 2 & $3 d$ & $1,2 \mathrm{bc}$ & $9 d$ & $7 \mathrm{c}$ & $3,3 \mathrm{ab}$ & $0,4 \mathrm{~cd}$ & $88 \mathrm{~cd}$ & $6 \mathrm{bc}$ & $217 \mathrm{~d}$ & $17 \mathrm{c}$ & $17 \mathrm{c}$ \\
\hline 3 & $6 \mathrm{c}$ & $1,9 \mathrm{~b}$ & $17 \mathrm{c}$ & $13 \mathrm{~b}$ & $6,2 \mathrm{ab}$ & $0,6 \mathrm{bc}$ & $169 \mathrm{c}$ & $14 \mathrm{~b}$ & $448 \mathrm{c}$ & $34 \mathrm{~b}$ & $30 \mathrm{~b}$ \\
\hline 4 & $11 \mathrm{~b}$ & $3,4 \mathrm{a}$ & $30 \mathrm{~b}$ & $25 \mathrm{a}$ & $5,9 \mathrm{ab}$ & $1,0 \mathrm{~b}$ & $339 \mathrm{~b}$ & $27 \mathrm{a}$ & $836 \mathrm{~b}$ & $48 \mathrm{a}$ & $38 \mathrm{~b}$ \\
\hline 5 & $20 \mathrm{a}$ & $4,0 \mathrm{a}$ & $36 \mathrm{a}$ & $29 \mathrm{a}$ & $8,7 \mathrm{a}$ & $1,4 \mathrm{a}$ & $441 \mathrm{a}$ & $28 \mathrm{a}$ & $1033 \mathrm{a}$ & $61 \mathrm{a}$ & $74 \mathrm{a}$ \\
\hline
\end{tabular}

As médias com as mesmas letras dentro de cada espécie não diferem estatisticamente (teste deTukey, $\mathrm{P}>0,05$ ).

Means with the same letter are not significantly different within each species (Tukey test, P>0,05).

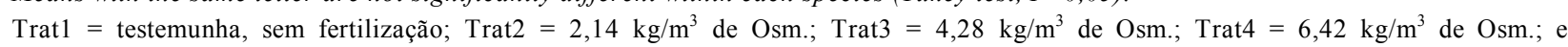
Trat5 = fertilização convencional.

maior dose de FLC. Quanto ao Peltophorum dubium, os valores de conteúdo da parte aérea das mudas nos tratamentos com maiores doses de FLC não diferiram para os elementos P, K, Ca, Mg, S, Cu, Zn e B. Para o Eucalyptus grandis, esta tendência de valores não-significativos de conteúdo da parte aérea das mudas ocorreu entre o tratamento com maior dose de FLC e o convencional, para os elementos $\mathrm{P}, \mathrm{Ca}, \mathrm{Mg}, \mathrm{Cu}$ e $\mathrm{Zn}$.

A maior concentração de nutrientes da parte aérea das mudas na testemunha (sem adubação), para os elementos N, P, Ca e Mg, para a Guazuma ulmifolia e P para o Peltophorum dubium, em relação a pelo menos um tratamento de adubação (Quadro 3), denota um efeito de diluição destes, como atestam os maiores valores de conteúdo desses elementos nos tratamentos adubados (Quadro 4), o que resulta em maior crescimento das mudas (Quadro 2). Por outro lado, a maior concentração de $\mathrm{N}$ na parte aérea das mudas de Eucalyptus grandis no tratamento convencional (Quadro 3) parece refletir parcialmente um consumo de luxo, pois a altura das mudas nesse tratamento foi maior (Quadro 2).
Visualizando a razão entre biomassa de raiz e parte aérea (R/P), para a Guazuma ulmifolia, o Peltophorum dubium e o Eucalyptus grandis (Quadro 2), e o seu conteúdo de $\mathrm{N}$ da parte aérea (Quadro 4), observa-se uma correlação negativa entre a razão $\mathrm{R} / \mathrm{P}$ e o conteúdo de $\mathrm{N}$ na parte aérea das mudas. Este fato foi observado também por Worral et al. (1987) em mudas de 16 espécies arbóreas ornamentais, por Cromer \& Jarvis (1990) para mudas de Eucalyptus grandis e por Ogbonnaya \& Kinako (1993) para mudas de Gmelina arborea. Um outro fator que pode ter colaborado, provavelmente em menor escala, com uma menor razão R/P no tratamento convencional é a fonte de $\mathrm{N}$ utilizado (amoniacal). Nos tratamentos com FLC o $\mathrm{N}$ foi fornecido na forma nítrica e amoniacal. Grespan et al. (1998), em experimento com mudas de quatro espécies de Eucalyptus submetidas a diferentes relações amônio/nitrato, observaram que quando se elevou a proporção de nitrato no meio a relação entre raiz e parte aérea para estas quatro espécies aumentou. Por outro lado, a maior concentração de $\mathrm{N}$ na parte aérea das mudas no tratamento convencional para o Peltophorum dubium e o Eucalyptus grandis (Quadro 3) comparados 
aos tratamentos com FLC pode, em parte, ser causado pelo fornecimento de $\mathrm{NH}_{4}^{+}$naquele tratamento. Ogbonnaya \& Kinako (1993) observaram em mudas de Gmelina arborea que a concentração de $\mathrm{N}$ nas folhas foi maior com a fonte $\mathrm{NH}_{4}{ }^{+}$(sulfato de amônio) do que com nitrato de amônio.

A maior altura das mudas e biomassa seca da parte aérea (BSPA) no tratamento convencional (Quadro 2) para a Guazuma ulmifolia, o Peltophorum dubium e o Eucalyptus grandis pode ter sido devido à maior absorção de N, bem como ao fornecimento de micronutrientes, que só este tratamento teve. WRIGHT et al. (1999), em estudo de crescimento de nove espécies arbóreas, utilizando ou não calcário e micronutrientes em dois substratos de casca de Pinus taeda, com diferentes valores de $\mathrm{pH}$, observaram uma maior BSPA e altura das plantas em todas as espécies onde foram utilizados micronutrientes.

No tratamento convencional, caso tivesse sido aplicado K na fertilização de cobertura de maneira mais periódica, o desenvolvimento das raízes provavelmente teria sido maior, podendo refletir em maior razão entre biomassa seca da raiz e parte aérea, ou não, no caso dessas duas partes terem crescimentos proporcionais. Xu et al. (1992), em experimento sobre a interação de K com a forma e a quantidade de N no crescimento de Zea mays, observaram, quando foram fornecidos $2 \mathrm{mmol} / \mathrm{l}$ de $\mathrm{N}$ e a quantidade de $\mathrm{K}$ variou, para se obterem três razões $\mathrm{N} / \mathrm{K}$ $\left(4,1 \mathrm{e}^{1 / 4}\right)$, que as duas menores razões propiciaram mudas com maior desenvolvimento das raízes e parte aérea. Concomitantemente, Moraes Neto et al. (2000), em experimento com seis espécies arbóreas nativas, produzidas em tubetes de $50 \mathrm{~cm}^{3}$ de capacidade e com fertilização de base similar à do presente experimento, porém utilizando como fertilização de cobertura K aplicado a cada quatro semanas durante a fase experimental, além de sulfato de amônio aplicado a cada duas semanas, constataram que as mudas de Guazuma ulmifolia e Peltophorum dubium apresentaram, a pleno sol, razões $\mathrm{R} / \mathrm{P}$ de 0,72 e 0,36 , respectivamente, enquanto no presente experimento as razões foram de 0,37 e 0,17 , respectivamente, devendo-se ressaltar que as mudas do primeiro alcançaram um bom crescimento.

Dentre os tratamentos que poderiam suscitar dúvida de qual seria o mais apropriado para a Guazuma ulmifolia, o Peltophorum dubium e o Eucalyptus grandis, mencionam-se o tratamento com maior dose de $\operatorname{FLC}\left(6,42 \mathrm{~kg} / \mathrm{m}^{3}\right)$ e o tratamento convencional. O tratamento convencional produziu maior crescimento em altura para a
Guazuma ulmifolia e o Peltophorum dubium, contudo nas duas primeiras espécies a razão entre biomassa seca da raiz e parte aérea $(\mathrm{R} / \mathrm{P})$ foi aproximadamente duas vezes inferior para o tratamento convencional quando comparado com o tratamento com maior dose de FLC (Quadro 2). Este fato, juntamente com um bom crescimento das plantas obtido pelo tratamento com maior dose de FLC para estas duas espécies, evidencia que seria mais prudente optar por este tratamento em relação ao convencional. Para o Eucalyptus grandis parece ser também indicado o tratamento com maior dose de FLC, pois as mudas nesta situação mostraram aspecto mais rústico (maior rigidez do caule), enquanto as plantas submetidas ao tratamento convencional mostraram-se mais tenras (menor rigidez do caule).

No Quadro 2 pode-se constatar que o Calycophyllum spruceanum e Pinus caribaea var. caribaea, onde foram aplicadas as maiores doses de FLC, resultou em plantas com maior altura e biomassa seca total do que as dos demais tratamentos para a primeira espécie, e valores (desses mesmos parâmetros) inferiores ao tratamento convencional para a segunda espécie. Contudo, as dimensões diminutas alcançadas por essas duas espécies permite inferir que os tratamentos só com FLC não seriam recomendáveis, pois os nutrientes podem ter sido lixiviados, devido ao fato de o seu tempo de liberação ser de três a quatro meses, o que coincide com o período experimental de 125 dias. Por outro lado, se houver adubação de cobertura complementar para os tratamentos com FLC, as plantas poderão obter um tamanho adequado para serem levadas ao campo.

Não se pode deduzir, até os 125 dias após a semeadura, se compensa utilizar o tratamento convencional para as espécies C. spruceanum e $P$. caribaea var. caribaea. Contudo, se forem aplicadas adubações de cobertura usando $\mathrm{N}$ e $\mathrm{K}$ de forma periódica, possivelmente sejam obtidas mudas de qualidade satisfatória.

\section{CONCLUSÕES}

- Para produção de mudas de Guazuma ulmifolia, Peltophorum dubium e Eucalyptus grandis, recomendase a utilização de $6,42 \mathrm{~kg}$ de Osmocote ${ }^{\circledR} 14-14-14$ por $\mathrm{m}^{3}$ de substrato (tratamento 4); e

- até os 125 dias após a semeadura, nenhum dos tratamentos usados foi suficiente para as mudas de Calycophyllum spruceanum e Pinus caribaea var. 
caribaea atingirem tamanho adequado para serem enviadas ao campo.

\section{REFERÊNCIAS BIBLIOGRÁFICAS}

BUDOWSKI, G. Distribution of tropical American rain forest species in the light of successional processes. Turrialba, v. 15, p. 40-42, 1965.

COLE, J. C.; DOLE, J. M. Temperature and phosphorus source affect phosphorus retention by a pine bark-based container medium. HortScience, v. 32, n. 2, p. 236-240, 1997.

CONOVER, C. A.; POOLE, R. T. Influence of shade and fertilizer source and level on growth, quality and foliar content of Philodendron oxycardium Schott. Journal of American Society Horticultural Science, v. 99, n. 2, p. $150-152,1974$.

CROMER, R. N.; JARVIS, P. G. Growth and biomass partitioning in Eucalyptus grandis seedlings in response to nitrogen supply. Australian Journal of Botany, v. 30, p. 265-278, 1990.

GONÇALVES, J. L. M.; POGGIANI, F. Substratos para produção de mudas florestais. In: CONGRESSO LATINO AMERICANO DE CIÊNCIA DO SOLO, 13., 1996, Águas de Lindóia-SP. Anais... Águas de Lindóia: USP-ESALQ/ SBCS/CEA/SLACS/SBM, 1996. CD-ROM.

GRESPAN, S. L.; DIAS, L. E.; NOVAIS, R. F. Crescimento e parâmetros cinéticos de absorção de amônio e nitrato por mudas de Eucalyptus spp. submetidas a diferentes relações amônio/nitrato na presença e ausência de fósforo. Revista Brasileira de Ciencia do Solo, v. 22, n. 4, p. 667-674, 1998.

HUETT, D. O.; MORRIS, S. C. Fertiliser use efficiency by containerised nursery plants. 3 . Effect of heavy leaching and damaged fertiliser prills on plant growth, nutrient uptake, and nutrient loss. Australian Journal of Agriculture Research, v. 50, n. 2, p. 217-222, 1999.

MORAES NETO, S. P. et al. Crescimento de mudas de algumas espécies arbóreas que ocorrem na Mata Atlântica, em função do nível de luminosidade. Revista Árvore, v. 24 , n. 1 , p. $35-45,2000$.
OGBONNAYA, C. I.; KINAKO, P. D. S. Growth and nutrition of Gmelina arborea Roxb. seedlings fertilizes with four sources of nitrogen on a latosolic soil. Tree Physiology, v. 12, n. 3, p. 291-299, 1993.

SARRUGE, J. R.; HAAG, H. P. Análise química em plantas. Piracicaba: Escola Superior de Agricultura Luiz de Queiroz, 1974. 56 p.

SAS INSTITUTE. SAS procedures guide, version 6. 4.ed. Cary, North Carolina, 1990.

STEEL, R. D.; TORRIE, J. H. Principles and procedures of statistics: a biometrical approach. 2.ed. New York: McGraw Hill, 1980. 633 p.

TRENKEL, M. E. Improving fertilizer use efficiency: controlled-release and stabilized fertilizers in agriculture. Paris: International Fertilizer Industry Association, 1997. $151 \mathrm{p}$.

VALERI, S. V.; CORRADINI. L. Fertilização em viveiros para produção de mudas de Eucalyptus e Pinus. In: GONÇALVES, J. L. M.; BENEDETTI, V. Nutrição e fertilização florestal. Piracicaba: Instituto de Pesquisa Florestal, 2000. Capítulo 6, p. 167-190.

XU, Q. F.; TSAI, C. L.; TSAI, C. Y. Interaction of potassium with the form and amount of nitrogen nutrition on growth and nitrogen uptake of maize. Journal of Plant Nutrition, v. 15, n. 1, p. 23-33, 1992.

WORRAL, R. J.; LAMONT, G. P.; OÇONNELL, M. A. The growth response of container-grown woody ornamentals to controlled-release fertilizers. Scientia Horticulturae, v. 32, n. 3/4, p. 275-286, 1987.

WRIGHT, A. N. et al. Preplant lime and micronutrient amendments to pine bark affect growth of seedlings of nine container-grown tree species. HortScience, v. 34, n. 4, p. 669-673, 1999.

YAGÜE, J. L. F. El suelo y los fertilizantes. Ministerio de agricultura, pesca y alimentacion. Instituto Nacional de reforma y desarrollo agrario. Ediciones Mundi-Prensa, 1994. $327 \mathrm{p}$. 